\title{
Computing in the Past with Forward Integration
}

\author{
C. W. Gear*and Ioannis G. Kevrekidis ${ }^{\dagger}$ \\ July 17, 2003
}

\begin{abstract}
Using existing, forward-in-time integration schemes, we demonstrate that under certain conditions it is possible to compute the solution at earlier times, even in the presence of stiffness (for which reverse integration is unstable). This technique can be used when a reverse integrator is not available - for example, when all one has is a forward-in-time legacy code. It can also be used for the "reverse coarse" integration of the macroscopic closure of a system defined by a microscopic model which itself is naturally forward in time (e.g. a particle model of a gas). The method proposed has stability properties that enable it to converge to stationary points of unstable stiff systems.
\end{abstract}

Keywords Reverse Integration, stationary points, differential equations.

\section{Introduction}

We consider the problem of computing a solution of

$$
\frac{d y}{d t}=f(y)
$$

"backward" in time in various situations in which direct numerical integration in reverse time ( $t$ decreasing) is not possible. Here $f$ is $\Re^{n} \mapsto \Re^{n}$. For example, we may have access only to a legacy code that computes $y(t+\Delta t)$ given $y(t)$ with positive $\Delta t$ not under our control; alternatively, eq. (1) might be the unknown closure ("coarse equation") of a microscopically-defined system which is inherently uni-directional in time.

In an earlier paper ([2]) we considered projective methods for stiff problems with gaps in their spectra. In the projective method, a numerical solution is computed at a sequence of relatively closely spaced points in time using a conventional integrator with small time steps, and then a "giant" step is taken using polynomial extrapolation from the last few of the points computed in the small step integration. This latter process is called the

*NEC Research Institute (Retired) \& Department of Chemical Engineering, Princeton University

${ }^{\dagger}$ Department of Chemical Engineering, PACM, \& Mathematics, Princeton University 
outer integrator and the giant step is called the projective step. It was taken forward in time. The small step integrations stabilized the fast ("strongly stable") components; the large, projective step had a stability region associated with an explicit, large step method that was stable for slowly damped components. The combined method had a stability region for linear problems that consisted of two subregions, one that caused damping of the fast components and one that caused damping of the slow. In this paper we take the large step in the reverse direction. The effect is that the small forward steps lead to the method being stable for eigenvalues corresponding to strongly damped components with time constants of the order of the small step, while the large backwards step adds a stability region corresponding to the large, explicit step, that is, a region that damps small positive eigenvalues.

In the next section we will briefly analyze the linear stability properties of these methods, and in the third section we will give an illustrative example. In the following two sections we will discuss the application of the method to computing the slowly changing component of forwardly unstable systems, as well as to unstable stationary points.

\section{Analysis}

We will analyze the simplest of these methods following the technique used in [2]. The reverse projective integration step we will discuss here consists of $k+1$ inner integration steps of size $h$ forward from $t_{n}$ to $t_{n+k+1}$, followed by one projective step of size $-M h$ to arrive at $t_{k+1-M}$. The projective step takes the form

$$
y_{k+1-M}^{p}=M y_{k}-(M-1) y_{k+1}
$$

Writing $m=M-k-1$ and one step of the inner integrator as $y_{n+1}=\phi\left(y_{n}\right)$ we see that, starting from an initial value $y_{0}$ the method is given by

$$
y_{-m(q+1)}=y_{-m q+k+1}-M\left(y_{-m q+k+1}-y_{-m q+k}\right)
$$

where

$$
y_{-m q+j+1}=\phi\left(y_{-m q+j}\right), j=0, \cdots, k
$$

In eq. (2) we see that $\left(y_{-m q+k+1}-y_{-m q+k}\right) / h$ is functioning as an estimate of the derivative at $t_{-m q+k+1}$.

As in [2] we assume that the error amplification of an inner integrator step is $\rho(h \lambda)$. One could conceptually study the case where the inner integrator is perfect i.e., $\rho(h \lambda)=$ $e^{h \lambda}$. For our analysis, we need only assume that the inner integrator is at least first order accurate, so that we have

$$
\rho(h \lambda)=1+h \lambda+\mathrm{O}(h \lambda)^{2} .
$$

(If the inner integrator is the forward Euler method, the last term can be dropped.) We see that the reverse projective method has an error amplification $\sigma$ given by

$$
\sigma=\rho^{k}(M-(M-1) \rho) .
$$


As before, we define the stability region in the $\rho$-plane as the set of $\rho$ for which $\sigma$ is not outside the unit disk, and plot its boundary by finding the set of $\rho$ such that $|\sigma|=1$. Figure 1 shows the plots for $k=2$ and four different values of $M, 4,5,7$, and 11 . Note that, because the process goes forward $k+1=3$ steps before going backwards $M$ steps, the values actually correspond to net reverse steps of $h, 2 h, 4 h$, and $8 h$ respectively. The method is stable inside the regions shown. As $M$ gets large, these regions asymptotically tend to a pair of disks. One, centered at $1+1 / M$ and of radius $1 / M$, corresponds to the stability region of the forward Euler method because the reverse projective step is the equivalent of a forward Euler method in the reverse direction. The second is centered at the origin and has radius $M^{-1 / k}$. It represents the region where the damping of $\rho^{k}$ is sufficient to overcome the growth proportional to $M$. In other words, the regions are essentially similar to those for forward projective methods, except that the stability region due to the projective step corresponds to $h \lambda$ in the positive half plane since that step is taken in the reverse direction.

In the example presented in the next section, we will use a method similar to the 2nd order Adams-Bashforth method as the outer (reverse projective) integrator. This corresponds to the integration sequence

$$
y_{-m(q+1)}=y_{-m q+k+1}+\beta_{1}(M)\left(y_{-m q+k+1}-y_{-m q+k}\right)+\beta_{2}(M)\left(y_{-m(q-1)+k+1}-y_{-m(q-1)+k}\right)
$$

coupled with eq. (3) where

$$
\beta_{1}(M)=-\frac{M}{2 m}(M-1+2 m), \quad \beta_{2}(M)=\frac{M(M-1)}{2 m}, \text { and } m=M-k-1 .
$$

Its stability region is similar, namely it is stable near the origin of the $\rho$-plane and in a region corresponding to the stability region of the reverse Adams Bashforth method. The stability region for the case $k=2, M=6$ is shown in 2 . The region in the right-half plane corresponds to the stability region of the Adams-Bashforth method of order 2 in the reverse direction.

\section{An example}

We consider a set of three coupled nonlinear ODEs that constitute a simplified model of the catalytic $C O$ oxidation reaction in the presence of an inert species (see [5]). The mean field evolution equations for this simplified mechanism, taking into account adsorption (dissociative for the oxygen), desorption (negligible for the oxygen) and second order surface reaction are

$$
\begin{gathered}
\frac{d \theta_{A}}{d t}=\alpha \theta_{*}-\gamma \theta_{A}-4 k_{r} \theta_{A} \theta_{B} \\
\frac{d \theta_{B}}{d t}=2 \beta \theta_{*}^{2}-4 k_{r} \theta_{A} \theta_{B}
\end{gathered}
$$




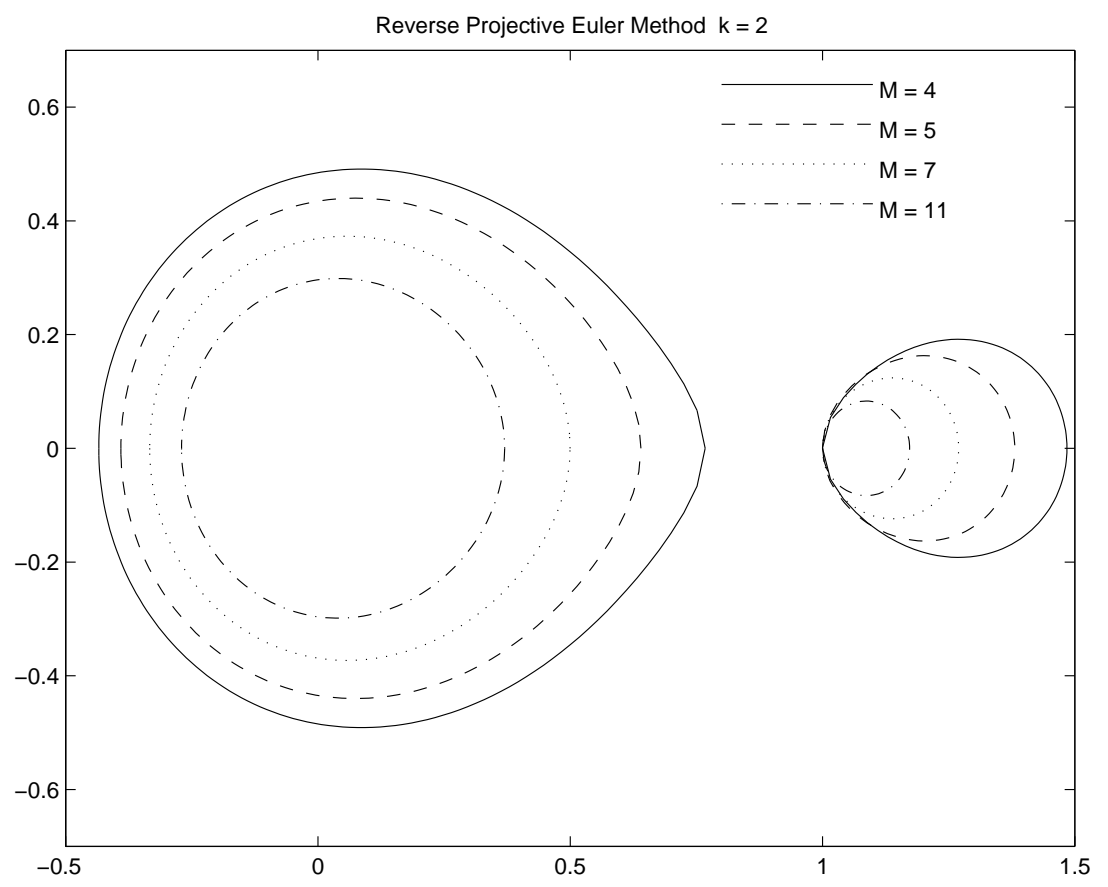

Figure 1: Stability Regions for the Reverse Projective Method with First-Order Outer Integrator.

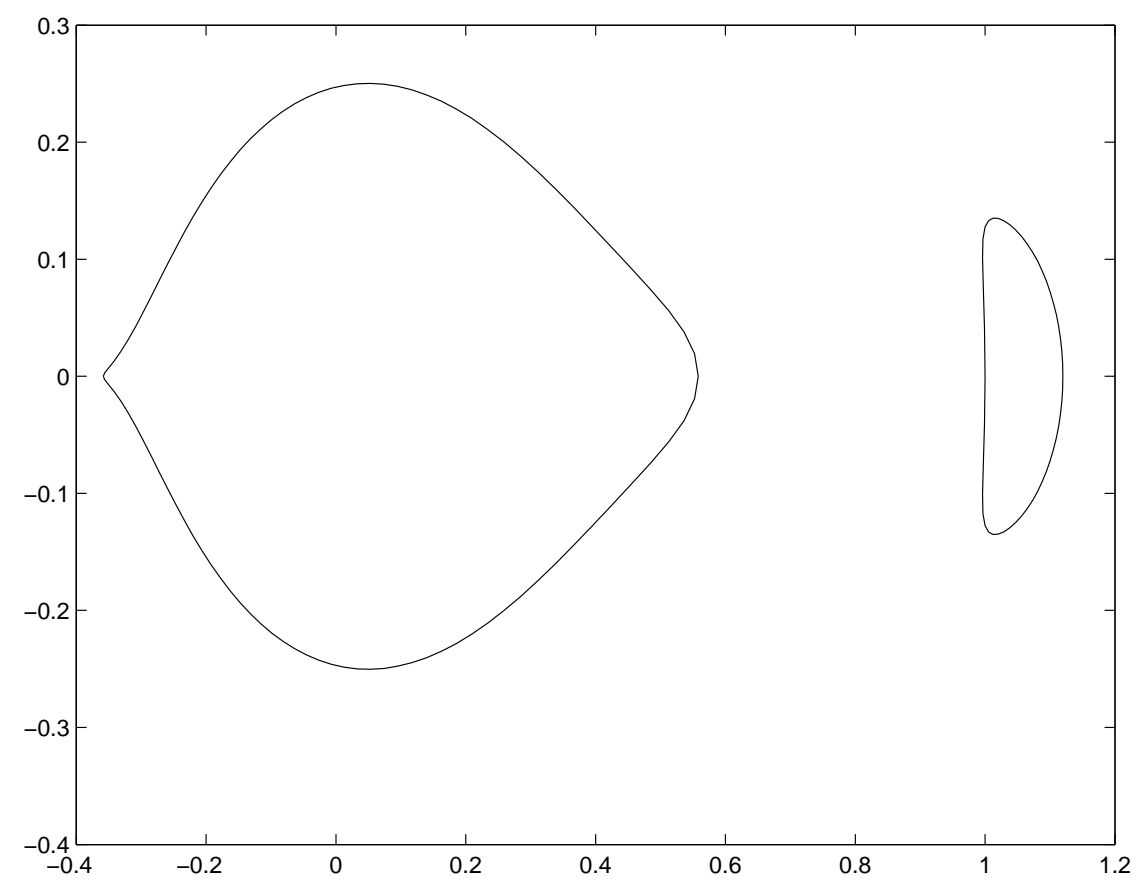

Figure 2: Stability Regions for the Reverse Projective Method with 2nd order AdamsBashforth Outer Integrator. $k=2$ and $M=6$. 


$$
\frac{d \theta_{C}}{d t}=\mu \theta_{*}-\eta \theta_{C}
$$

The fractional coverages of the surface by $C O, O$ and the inert species $C$ are $\theta_{A}, \theta_{B}$ and $\theta_{C}$ respectively, while the empty site fraction is $\theta_{*}=1-\theta_{A}-\theta_{B}-\theta_{C}$. The physical meaning of the various parameters and kinetic constants can be found in [5], which also contains a bifurcation diagram for this model as a function of the gas phase partial pressure of oxygen, $\beta$.

In a realistic situation we would be interested in numerically analyzing a much more detailed model than this, for example, a stochastic model based on the Gillespie algorithm as discussed in [7]. We use this simplified version for expository purposes since it has similar behavior. If we used the stochastic model we would only be able to integrate it in the forward direction.

The parameter values used for this case were: $\alpha=1.6, \beta=20.8, \gamma=0.04, \mu=$ $0.36, \eta=0.016$, and $k_{r}=1$. With these values, the system possesses an unstable steady state, surrounded by an attracting limit cycle. Transients are fairly strongly attracted to this stable limit cycle. For example, if we were to start from the initial condition $(0.2776$, 0.0324, 0.6605) which lies close to the single, unstable saddle point, the trajectory will reach the limit cycle to plot accuracy in about 700 time units, and along that trajectory one eigenvalue of the local linearization of the differential equation lies between -6.47 and -5.08 while the other two had real parts between -0.05 and +0.21 and imaginary parts that were less than 0.26 in magnitude. Clearly direct integration in the reverse direction would be extremely unstable because of the eigenvalue near -6 .

Starting from a point near the limit cycle, $\theta=(0.342778296,0.019029657,0.61305464)$, we used reverse projective integration. First we integrated in the forward direction with the Euler method using step size 0.16 for 3 steps and used the last two steps to estimate the chord slope. Then eq. (4) was applied with $M=6$ to get an effective reverse step size of $-3 h=-0.48$. Any component corresponding to the large negative eigenvalue at about -5.5 is quickly damped, so the integration could proceed in the reverse direction until the trajectory approached the neighborhood of the saddle point at $\theta=(0.278291262,0.032174358,0.660192491)$.

Figure 3 shows the components of $\theta$ as a function of time. Figure (4) shows the projected phase-plane plot of $\theta_{A}$ versus $\theta_{C}$. These were integrated only until they were within about $10^{-3}$ of the saddle point for two reasons. First, the behavior at earlier times (later in the integration!) is uninteresting; second, we wished to demonstrate that the reverse integration captures what would have been found from a forward integration. After the reverse integration was stopped at $t=-600$, a forward integration was performed to $t=0$, the original starting point. The plots shown consists of two curves for each segment, one for the reverse integration (solid line) and one for the forward (dashed line), but because they are so close, the two curves are almost indistinguishable. For this reason we also show a blow-up of the region around $t=0$ where it can be seen that the curves differ visually by very little. In fact, the max norm difference between the forward 

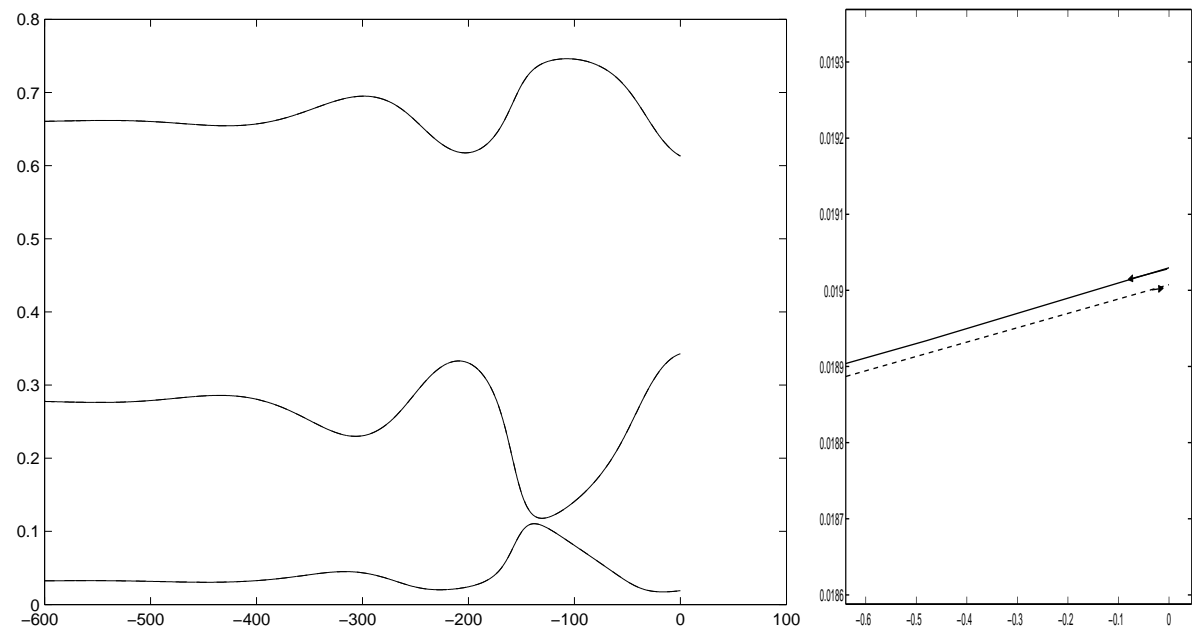

Figure 3: Reverse integration of eq. (5) from $t=0$ to $t=-600$ (solid line) and then forward (dashed) to $t=0$. Components are $\theta_{C}, \theta_{A}$, and $\theta_{B}$ from top to bottom. Right panel shows $\theta_{B}$ near $t=0$.
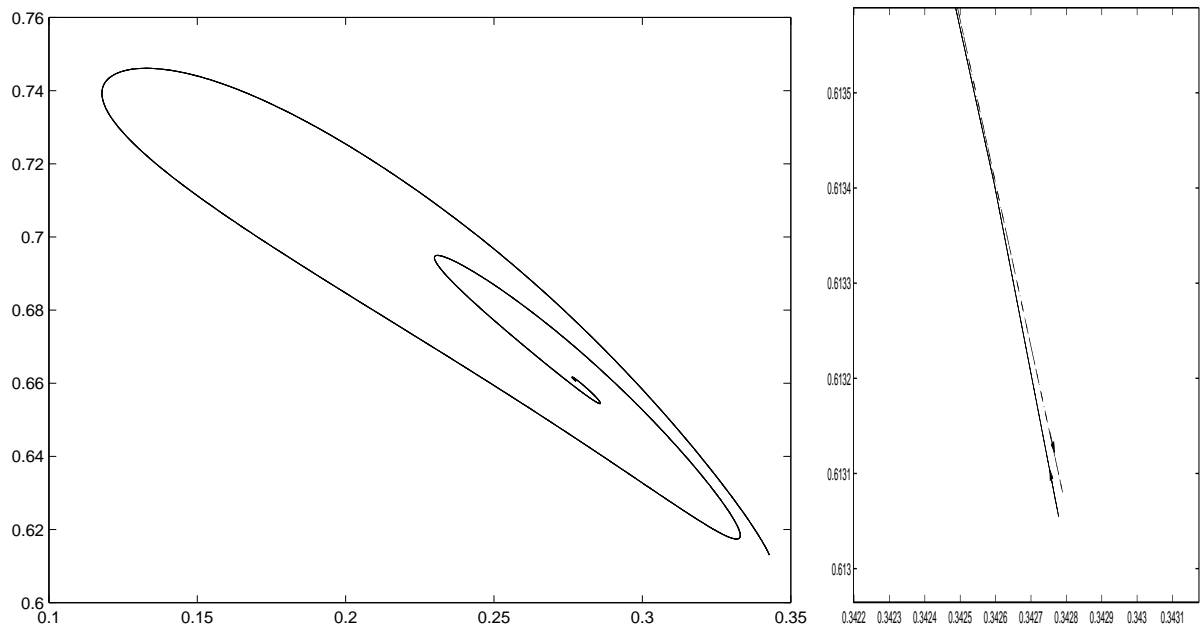

Figure 4: $\theta_{A}-\theta_{C}$ projected phase-plane plot of transient in 3. Right panel is enlargement near $t=0$. 
and backward solutions is $3.3 \times 10^{-4}$. If these equations were integrated in the reverse direction with a standard integrator the result would be numerical overflow since over a range of -600 the eigenvalue of about -5.7 causes an amplification of its eigencomponent by about $e^{3420}$ which is approximately $10^{1485}$. Unless one had starting values to that accuracy and worked to a precision greater than about 4950 bits, amplified roundoff errors would dominate the solution!

\section{Projective Integration of Forwardly Unstable sys- tems}

While we have introduced this compound method (forward inner integration plus a reverse projective step) for the reverse integration when direct integration in the reverse direction is impractical, it can also be used to compute the sub-dominant components of a forwardly unstable system, that is, the slow components that do not blow up in forward time. For this application we must be able to execute the inner integrator in the reverse time direction, and then take the projective step in the forward direction. The stability regions will simply be the mirror images in the $\rho=1$ line of those shown in Figs (1) and (2).

It is also interesting that for problems with only unstable eigendirections, separated in fast and slow components, this "flipped reverse" scheme may help track the slow unstable submanifold of the higher-dimensional unstable manifold of the problem.

\section{$5 \quad$ Stationary Projective Methods}

In the above we have taken $M>k+1$ so that the net progress is negative in time. If we choose $M=k+1$ in eq. (2) the total step length of the reverse projective integration method will be zero; we will call this a stationary projective method. It has the curious stability region shown in Figure 5. It is stable along a section of the real axis that includes $\rho=1$ (which is $h \lambda=0$ ). The boundary crosses itself at $\rho=1$. The intersection is at right angles because in the neighborhood of $\rho=1$ simple algebra shows that $\sigma$ is given by

$$
\sigma=1-k(k+1) / 2(\rho-1)^{2}-k^{2}(k-1) / 2(\rho-1)^{3}
$$

so the stability in the neighborhood of $\rho=1$ is equivalent to requiring that

$$
\operatorname{Real}\left((\rho-1)^{2}\right) \geq 0
$$

which happens when $\operatorname{Arg}(\rho) \in[-\pi / 4, \pi / 4]$ or $[3 \pi / 4,5 \pi / 4]$. The form of eq. (6) arises because the first-order accuracy of the method for a net step size of length zero means that

$$
\sigma(0 \lambda)=1+0 \lambda+\mathrm{O}(h \lambda)^{2}=1+\mathrm{O}(\rho-1)^{2}
$$


This can be used to find saddle stationary points provided that the arguments of the complex eigenvalues are within $\pi / 4$ of the negative or positive real axes. When there are only a few eigenvalues close to the imaginary axis, whether in the negative or positive half plane, other fixed point algorithms, based on timestepping and subspace estimation, such as RPM [8] can be used to find the stationary points effectively. If many eigenvalues exist close to (on either side of) the imaginary axis, estimating a high dimensional slow subspace may become impractical. As an example, the following shift of the reaction-diffusion equation,

$$
u_{t}=-u^{3}+3 u^{2} q(x)-3 u q^{2}(x)+q^{3}(x)+u+\nu u_{x x}-\nu q_{x x}(x),
$$

was solved with $q(x)=0.05 x(\pi-x)$ and boundary conditions $u(0, t)=u(\pi, t)=0$. When $\nu=10^{-2}$ it has 10 eigenvalues in the positive half plane near the zero stationary saddle point, $u=q(x)$. If a standard finite difference approximation is applied to this with 30 discretization points, the remaining 20 eigenvalues will be in the negative half plane.

Starting from $u(x, 0)=0$ and using $k=5$ and $M=6$ in the procedure, the saddle point $q(x)$ was found in 364 explicit steps to $10^{-5} \mathrm{~L} 1$ norm accuracy. (Other stationary points exist for this problem. Starting from different initial conditions may converge to such points.)

At the stationary point of interest, the 30 eigenvalues of the linearized operator range from

$$
(-2.90, \cdots,-0.279,-0.098+0.075,+0.237 \cdots,+0.978),
$$

where we have shown the smallest, the 19th, 20th, 21st, and 22nd (which span zero), and the largest eigenvalues. When a Forward Euler inner integrator is used with step size $h=0.25$ the eigenvalues of the Euler process become

$$
(+0.276, \cdots,+0.930,+0.976,+1.019,+1.060, \cdots,+1.245)
$$

which is clearly unstable (as is to be expected because of the 10 positive eigenvalues). However, the eigenvalue of the stationary projective process are

$$
(+0.007, \cdots,+0.940,+0.992,+0.995,+0.940, \cdots,-0.666) .
$$

In other words, the process maps those real eigenvalues outside the unit circle inside, while leaving those real eigenvalues initially inside still inside, thus making the iteration stable.

The convergence is slow because the 20th and 21st eigenvalues are very close to the unit circle. Various forms of iteration acceleration, such as Newton-Krylov (e.g., the Shroff-Keller method [8]) could be conceivably applied to this stationary integration process to speed it up. 


\section{Comments}

The reverse projective procedure we just outlined will, under the conditions discussed, be an "on manifold" reverse time integration scheme if the forward in time dynamics possess a separation of time scales globally, and an attracting, forward-invariant, low-dimensional slow manifold exists. That is, the regularizing action of the forward integration allows us to follow the on-manifold well-behaved trajectories backward in time. This may then provide a meaningful -and very simple to implement- way of regularizing the reverse, "on the slow manifold", dynamics of stiff sets of ODEs and even discretizations of dissipative PDEs. For example, in contexts where a low-dimensional inertial manifold exists for a dissipative PDE [10], our superstructure enables a direct simulation of an accurate discretization of the PDE to approximately follow backward trajectories on the inertial manifold without ever having to explicitly derive an inertial form (or approximate inertial form). This might be useful in certain inverse problems, as well as cases involving dynamic computations in an optimal control / optimization context, where direct reverse integration on the slow manifold of a highly dissipative problem may be otherwise very difficult or practically impossible.

In the spirit of the last observation, it is interesting to consider the implications of such a process for a meaningful reverse integration of systems described by microscopic/stochastic simulators. In many practically relevant cases, the coarse-grained behavior of such simulators can be described by the evolution in time of a few "master" moments of microscopically evolving distributions. The remaining, higher moments, become quickly slaved by forward simulation to the slow, master moments. We have recently proposed coarse projective integration schemes that use short bursts of appropriately initialized microscopic/stochastic simulation to estimate the time-derivative of the unavailable coarse equations for the master modes, and "project" these modes forward in time $[4,9,6]$. If the coarse projection is performed backward in time, the procedure will allow us to follow the regularized reverse time behavior of the coarse variables. This is done using the microscopic/stochastic forward-in-time simulator directly, circumventing the necessity of deriving an explicit macroscopic closure. It becomes therefore possible to use a forward-in-time molecular dynamics simulator to extract regularized reverse-time information of coarse system variables. We have already demonstrated the feasibility of this technique in the case of coarse molecular dynamics simulations of a dipeptide folding kinetics in water [6]. Coarse reverse integration allows us to use microscopic simulators to quickly escape free-energy minima, to converge on certain transition states (saddles on the free-energy surface) and, more generally, may enhance our ability to explore the structure of free energy surfaces.

Beyond the computation of reverse trajectories, reverse projective integration allows us to converge to saddle-type points whose linearization is characterized by a gap between strong stable modes and weak unstable ones. An existing forward simulation code will not in general converge to such points (the numerical trajectories will move away forward in 
time, and "explode away" backward in time). We can therefore think of our procedure as a computational superstructure that transforms a forward simulation code into a contraction mapping capable of converging to such saddle unstable points.

The stationary projective process described at the end permits the computation of saddle points when there is a gap between strongly stable components and weakly stable or unstable components. It can be related to consistent initialization algorithms for differential-algebraic equations [1].

In summary, the technique holds promise towards (a) the regularized, "on manifold" backward-in-time integration of certain dissipative PDEs possessing low-dimensional, exponentially attracting slow manifolds, which can be useful in several contexts, including control and optimization; the computation of unstable, saddle-type fixed points using existing simulators; and (c) the use of microscopic/stochastic simulators to track coarsegrained behavior backward in time, enhancing the ability to escape free energy minima and to locate saddle-type coarse-grained "transition states".

Acknowledgements. This work was partially supported by AFOSR (Dynamics and Control, Dr. B. King) and an NSF ITR grant. Discussions with Profs. P. G. Kevrekidis (UMass), Ju Li (OSU) and Dr. G. Hummer (NIH) are gratefully acknowledged.

\section{References}

[1] Brown P. N., Hindmarsh A. C. and Petzold L. R. (1998). Consistent initial condition calculation for differential-algebraic systems, SIAM J. Sci. Comput., 19, pp.14951512

[2] Gear, C. W. and Kevrekidis, I. G.,(2003) Projective Methods for Stiff Differential Equations: problems with gaps in their eigenvalue spectrum, SIAM J. Sci. Comp. 24(4) pp. 1091-1106.

[3] Gear, C. W. and Kevrekidis, I. G., (2003) Telescopic Projective Methods for Stiff Differential Equations, J. Comp. Phys. 187, 1, pp 95-109.

[4] Gear, C. W., Kevrekidis, I. G. and Theodoropoulos, K. (2002) Coarse Integration/Bifurcation Analysis via Microscopic Simulators: micro-Galerkin methods, Comp. Chem. Engng. 26 pp.941-963

[5] Makeev, A. G., Maroudas, D. and Kevrekidis I. G. (2002) Coarse stability and bifurcation analysis using stochastic simulators: kinetic Monte Carlo examples, $J$. Chem. Phys. 116(23) pp.10083-10091.

[6] Hummer, G. and Kevrekidis, I. G. (2003) Coarse molecular dynamics of a peptide fragment: free energy, kinetics and long time dynamics computations", J. Chem. Phys. 118(23) pp. 10762-10773. 
[7] Rico-Martinez, R., Gear, C. W. and Kevrekidis, I. G., (2003) Coarse Projective kMC Integration: Forward/Reverse Initial and Boundary Value Problems, submitted to J. Comp. Phys.; can be obtained as nlin.CG/0307016 at arXiv.org.

[8] Shroff, G.M. and Keller, H.B. (1993) Stabilization of unstable procedures: A recursive projection method, SIAM J. Numer. Anal. 30, 1099-1120.

[9] Siettos, C. I., Graham, M. D. and Kevrekidis, I. G. (2003) Coarse Brownian Dynamics Computations for Nematic Liquid Crystals" J. Chem. Phys. 118(22) pp.1014910157

[10] Témam, R. (1988) Infinite Dimensional Dynamical Systems in Mechanics and Physics Springer Verlag, NY. 


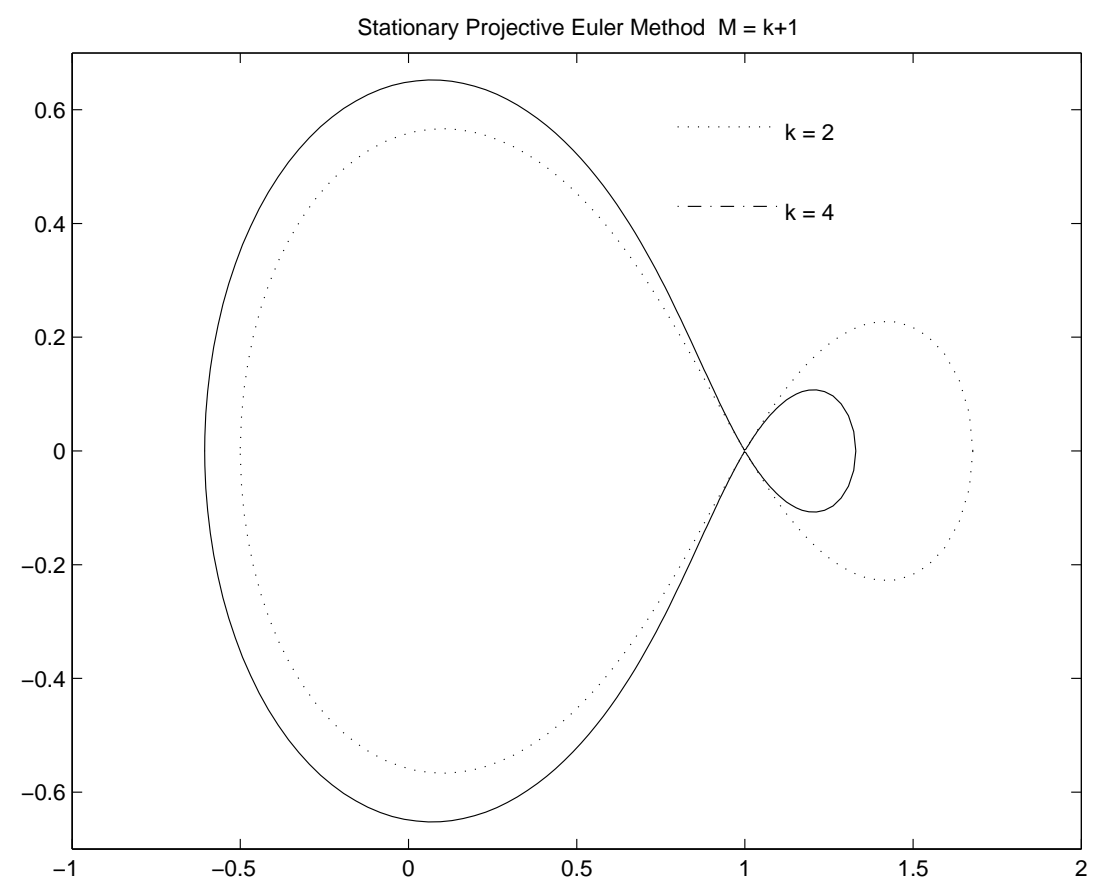

Figure 5: Stability Regions for Stationary Projective Methods. 